\title{
Diagnostic challenges in multiple system atrophy
}

This article was published in the following Dove Press journal:

Neuropsychiatric Disease and Treatment

\author{
Diana Obelieniene \\ Sandra Bauzaite' \\ Ilona Kulakiene ${ }^{2}$ \\ Evaldas Keleras ${ }^{2}$ \\ Indre Eitmonaite ${ }^{3}$ \\ Daiva Rastenyte' \\ 'Department of Neurology, \\ ${ }^{2}$ Department of Radiology, ${ }^{3}$ Medical \\ Academy, Lithuanian University of \\ Health Sciences, Kaunas, Lithuania
}

Correspondence: Diana Obelieniene Department of Neurology, Lithuanian University of Health Sciences, Eiveniu Street 2, Kaunas LT- 50I6I, Lithuania Email diana.obelieniene@kaunoklinikos.It

\begin{abstract}
Multiple system atrophy is a progressive neurodegenerative disorder that is characterized by autonomic failure, cerebellar ataxia and parkinsonism syndrome in various combinations. In spite of the presence of well-established clinical criteria for multiple system atrophy, ante-mortem diagnosis is difficult. In our case report, we present a 78-year-old female patient who presented with early progressive aphasia and severe autonomic dysfunction. Two years after appearance of the first symptoms, she fulfilled all the major criteria for probable multiple system atrophy with rapid progression. In addition, brain magnetic resonance imaging and fluorodeoxyglucose-positron emission tomography findings were more typical for progressive supranuclear palsy. Clinically differentiating multiple system atrophy from progressive supranuclear palsy and other similar neurodegenerative disorders may be challenging in all stages of the disease, especially with atypical disease presentation.
\end{abstract}

Keywords: multiple system atrophy, progressive aphasia, dementia, neurodegenerative diseases, progressive supranuclear palsy, FDG-PET, MRI, MRA

\section{Background}

Multiple system atrophy (MSA) is a sporadic, adult-onset (mean age at disease onset from 55 to 58 years) and progressive (mean disease duration from 7 to 9 years) neurodegenerative disorder that may manifest with diverse clinical features. ${ }^{1}$ Its classical clinical presentation is characterized by varying severity of parkinsonian features, cerebellar ataxia, autonomic failure, urogenital and corticospinal tract dysfunction. The disease frequently manifests with bladder dysfunction and erectile dysfunction in males and as orthostatic hypotension. Sometimes, dementia, depression and sleep disorders may occur. ${ }^{1}$ The prevalence of Rapid eye movement sleep Behavior Disorder (RBD) is significantly high ( $88 \%$ ) in MSA patients. RBD signs were present in about half of MSA patients before the onset of motor symptoms. ${ }^{2}$

Two phenotypes can be distinguished clinically: the parkinsonian (MSA-P) and the cerebellar type (MSA-C). Revised consensus criteria suggest three diagnostic categories of increasing certainty: possible, probable and definite. Definite diagnosis is confirmed by neuropathological examination. Autopsy studies revealed a wide range of diagnostic accuracy - between $29 \%$ and $86 \% .^{3,6}$ Difficulty in clinical diagnosis of MSA can also occur when MSA coexists with other neurodegenerative disease. The neuropathological hallmark of MSA is the presence of $\alpha$-synuclein immunoreactive cytoplasmic inclusions in neuroglia cells. ${ }^{4,9}$ A definite diagnosis of MSA is established when there is evidence of central nervous system $\alpha$-synuclein positive cytoplasmic inclusions in association with neurodegenerative changes, even in the absence of a clinical history of MSA. ${ }^{1}$ The importance of $\alpha$-synuclein immunohistochemistry is highlighted by the fact that other neurodegenerative diseases that overlap clinically with MSA, such as progressive supranuclear palsy (PSP), are also characterized by glial cytoplasmic inclusions that are instead positive for tau protein. 
The revised consensus criteria for the diagnosis of MSA have included magnetic resonance imaging (MRI) and fluorodeoxyglucose-positron emission tomography (FDG-PET) changes as supportive evidence for the diagnosis of possible MSA. ${ }^{1}$ The imaging features of possible MSA-P diagnosis include atrophy in the putamen, middle cerebellar peduncle, pons, cerebellum and hypometabolism in the putamen, brainstem or cerebellum. ${ }^{5}$ However, the diagnosis of MSA is based on clinical disease presentation and disease course. ${ }^{1}$

We present the case of a female patient with atypical MSA manifestation, lack of specific imaging features in the disease course and challenging differential diagnosis with similar neurodegenerative diseases.

\section{Case report}

A 78-year-old, right-handed Caucasian woman presented to our clinic with speech difficulties. About 4 months before admission, the patient noticed gradually increasing difficulty to find the right words and respond to questions, and her sentences became disjointed and less clear. Besides dysphasia and mild limb ataxia, her neurologic status was normal. She also had moderate orthostatic hypotension with no falls and mild urinary incontinence. Mental status was without cognitive impairment with a Mini-Mental State Examination (MMSE) score of 25. Polysomnography was not performed because the patient had no clinical signs of sleep-related disorder either in the disease manifestation or in the disease course. Her medical history was remarkable for hypothyroidism and episodic supraventricular tachycardia, and these disorders were treated with levothyroxine and beta blockers. There was nothing special in her family history. The first MRI was performed during the first hospitalization period. Mild brain atrophy and small vascular lesions were seen. Based on anamnesis, MRI, ultrasound investigations and laboratory findings, chronic cerebral ischemia was diagnosed.
The patient was admitted to our clinic for the second time only 2 years later. The clinical examination revealed global aphasia, bulbar syndrome, hypomimia, symmetrical bradykinesia with rigidity, hyperreflexia, bilateral Babinski sign, gait and limb ataxia and postural instability. The patient had not been able to walk without assistance for the last 3 months. She also had severe orthostatic hypotension, urinary incontinence and developed severe mental decline (MMSE score was 15). Blood tests were normal and included a basic metabolic profile, complete blood count, tests for thyroid and liver functioning, vitamin B1, vitamin B12 and folate levels and erythrocyte sedimentation rate. Cerebrospinal fluid examination in terms of cell count, glucose and protein revealed no abnormalities. She was given levodopa, prescribed by a local neurologist for parkinsonism syndrome treatment, for more than 3 months, but there was no response to the treatment.

MRI was repeated 29 months later. It revealed reduction of the anteroposterior diameter of midbrain at the superior collicular level from $15.5 \mathrm{~mm}$ on the first examination to $13.5 \mathrm{~mm}$ on follow-up images (Figure 1). Mild midbrain atrophy with concave upper surface was seen on sagittal images (the "hummingbird" or "penguin" sign), as shown in Figure 2. While these findings are suggestive of PSP, midbrain to pons area ratio on the midline sagittal plane was 0.24 , not showing rate reduction typical for PSP and remaining within normal range. Only minor putaminal hypointensity on T2-weighted images was noted (Figure 3A), which was not considered typical for MSA-P. There was also progression of generalized brain atrophy (Figures 2 and 3A, B), more prominent in the posterior frontal lobes.

For the differential diagnosis of neurodegenerative disease with rapidly progressive aphasia and dementia together with multisystem involvement of the central nervous system, FDG-PET was performed. Diffuse hypometabolism
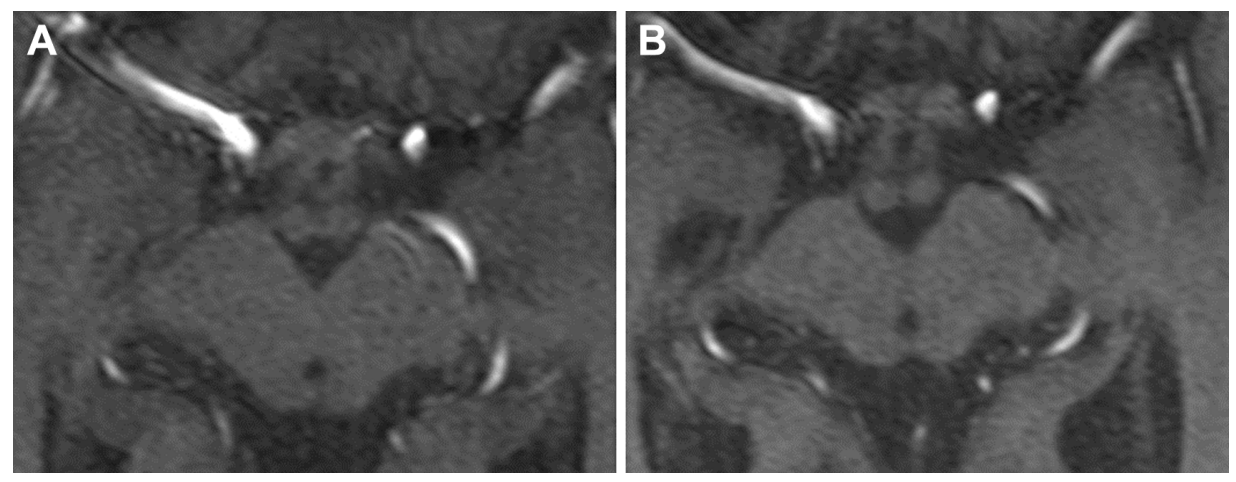

Figure I Multiplan reconstructions of 3D time-of-flight MRA images in transverse plane at the superior collicular level. (A) The first MR examination, (B) follow-up 2 years later. Note: Development of midbrain atrophy is seen on the follow-up image, with reduction of AP midbrain diameter.

Abbreviations: AP, anteroposterior; MR, magnetic resonance; MRA, magnetic resonance angiography. 


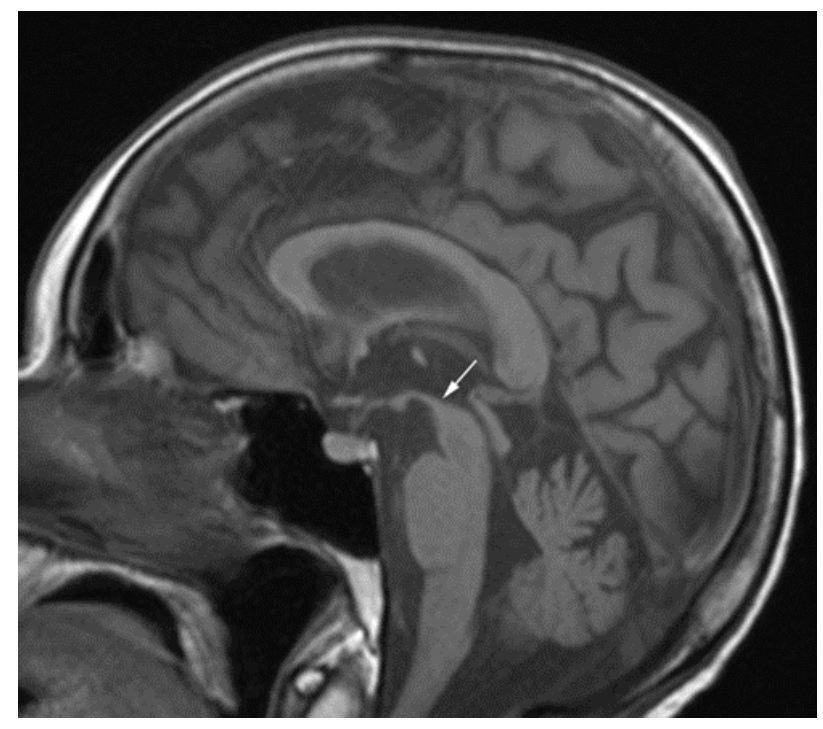

Figure 2 Sagittal spin-echo $\mathrm{Tl}$-weighted magnetic resonance images on follow-up examination show mild midbrain atrophy with "hummingbird" sign (arrow). Note: Development of generalized brain atrophy is also seen.

of both cerebral hemispheres was noted, which was especially pronounced in the middle and posterior part of the left frontal lobe. Small areas of hypometabolism were also present in the left lateral part of the temporal lobe and the left hemisphere of cerebellum. Diffusely decreased metabolism was reported in basal ganglia. Occipital lobes were less hypometabolic in comparison to the other brain areas (Figures 4 and 5). These findings were more typical for PSP than MSA. Despite the MRI and FDG-PET findings, the diagnosis of probable MSA was confirmed on the basis of neurologic clinical findings and disease course. The patient developed rapid disease progression and died 5 months later due to multiple complications and cardiopulmonary insufficiency. Neuropathological postmortem examination was not performed because the family members denied permission for autopsy.

\section{Ethical approval}

We confirm that written informed consent has been provided by the patient's legal caregiver to have the case details and any accompanying images published.

\section{Discussion}

Although definite diagnosis of the MSA can only be made by confirming neuropathological evidence, the diagnosis can be supported by clinical and imaging features. ${ }^{7,8}$ In this report, we present a woman with primary symptoms such as rapidly progressive aphasia, mild limb ataxia and autonomic dysfunction. After 2 years of the disease progression, she developed global aphasia, parkinsonism syndrome, cerebellar ataxia, pyramidal signs and severe mental decline. She had no response to dopaminergic treatment. Differential diagnosis was complicated because of atypical early disease presentation with dominant progressive aphasia and imaging findings, and was performed with frontotemporal dementia (FTD), corticobasal degeneration (CBD), dementia with Lewy bodies (DLB) and PSP.

FTD is characterized by personality and behavior changes in its early stage. ${ }^{6}$ In most cases, frontal and/or temporal lobe atrophy is seen on MRI. FTD patients can also develop muscle rigidity, tremor, akinesia and these symptoms can mimic MSA. ${ }^{3}$ In our case, some MRI and FDG-PET findings (generalized brain atrophy, more prominent in the posterior frontal lobe, and hypometabolism of both cerebral
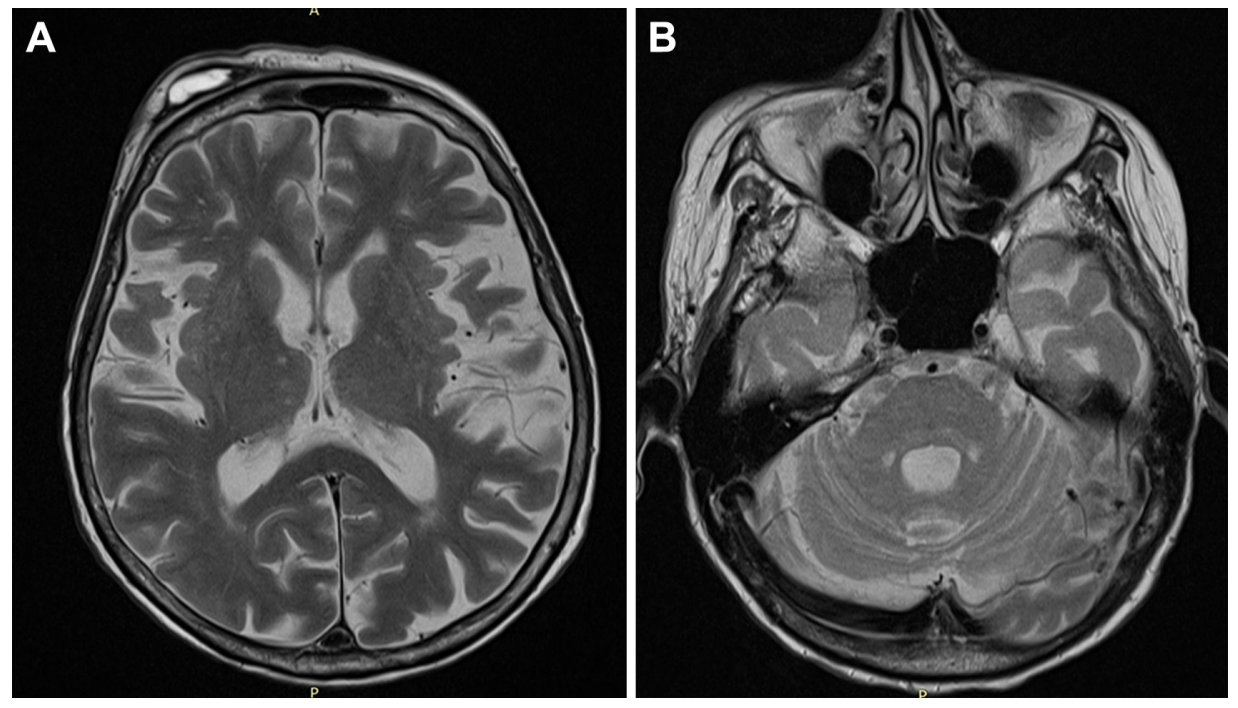

Figure 3 Transverse turbo spin-echo T2-weighted images on follow-up examination (A) at the basal ganglia and (B) at the pontine level.

Note: Minor bilateral putaminal hypointensity and generalized brain atrophy are seen. 


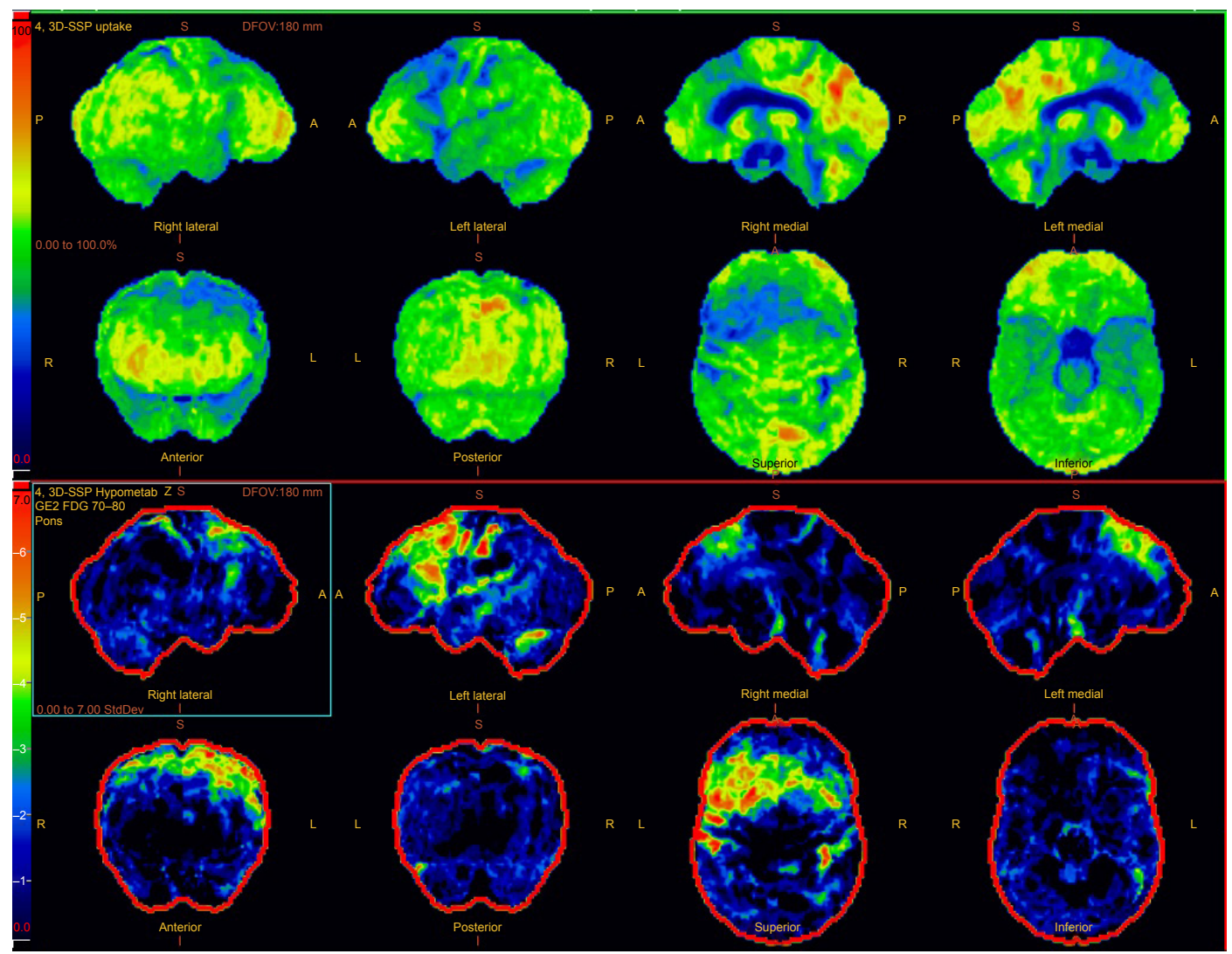

Figure 4 Brain three-dimensional stereotactic surface projection maps of positron emission tomography scans with activity maps in the top two rows and $\mathrm{z}$ maps showing deviation from a normal control cohort in the third and fourth rows.

hemispheres that was especially pronounced in the middle and posterior part of the left frontal lobe) could suggest FTD, although there were no mental and behavior changes in the early stage of the disease.

$\mathrm{CBD}$ is a disorder affecting the basal ganglia and the cerebral cortex, mainly the frontal and parietal lobes. Typically, it causes dementia, hallucinations and movement difficulties. MRI and FDG-PET can show asymmetric parietal atrophy and hypometabolism. ${ }^{8}$ Most patients with CBD develop progressive asymmetric rigidity in the limbs. In some cases, the legs may be affected before the arms. ${ }^{3}$ Our patient presented with mild limb ataxia, and revealed symmetrical bradykinesia with rigidity without apraxia after 2 years, which is more typical of MSA.

DLB, like MSA, is also characterized by deposits of degenerative $\alpha$-synuclein proteins (Lewy bodies). It is a form of parkinsonism in which cognitive impairment and hallucinations occur along with the classic parkinsonism signs of slow movement, rigid posture, unstable gait and tremor. ${ }^{3}$ If cognitive impairments precede movement disorder by at least 1 year, the diagnosis of DLB could be confirmed. If motor disturbance occurs before or concomitantly with cognitive impairment, the condition is usually classified as atypical parkinsonism with dementia. ${ }^{10,11}$ In our case, there were no early mental and parkinsonian symptoms, as well as the imaging features typical of DLB.

PSP is another disorder characterized by cognitive impairment, vertical supranuclear ophthalmoplegia, visual disturbance, extrapyramidal signs and a disturbance of gait resulting in early falls. PSP may manifest with different clinical features. MSA can be difficult to differentiate from PSP not only in the early stage, but also at late stages of the disease. Postural instability and limitation of vertical gaze are the early findings in PSP. Early falls and vertical gaze palsy without dysautonomia may differentiate PSP from MSA. The guidelines emphasized the importance of autonomic dysfunction by making this feature important for the diagnosis of probable MSA. ${ }^{1}$ Although most patients with PSP masquerading as MSA presented or developed cerebellar ataxia, the presence of prominent early cerebellar symptoms is an exclusion criterion for the clinical diagnosis of PSP. Severe cognitive decline is a supportive feature in the diagnosis of PSP. ${ }^{12}$ Although evident in both PSP and MSA, cognitive impairment is not a primary diagnostic criterion for 

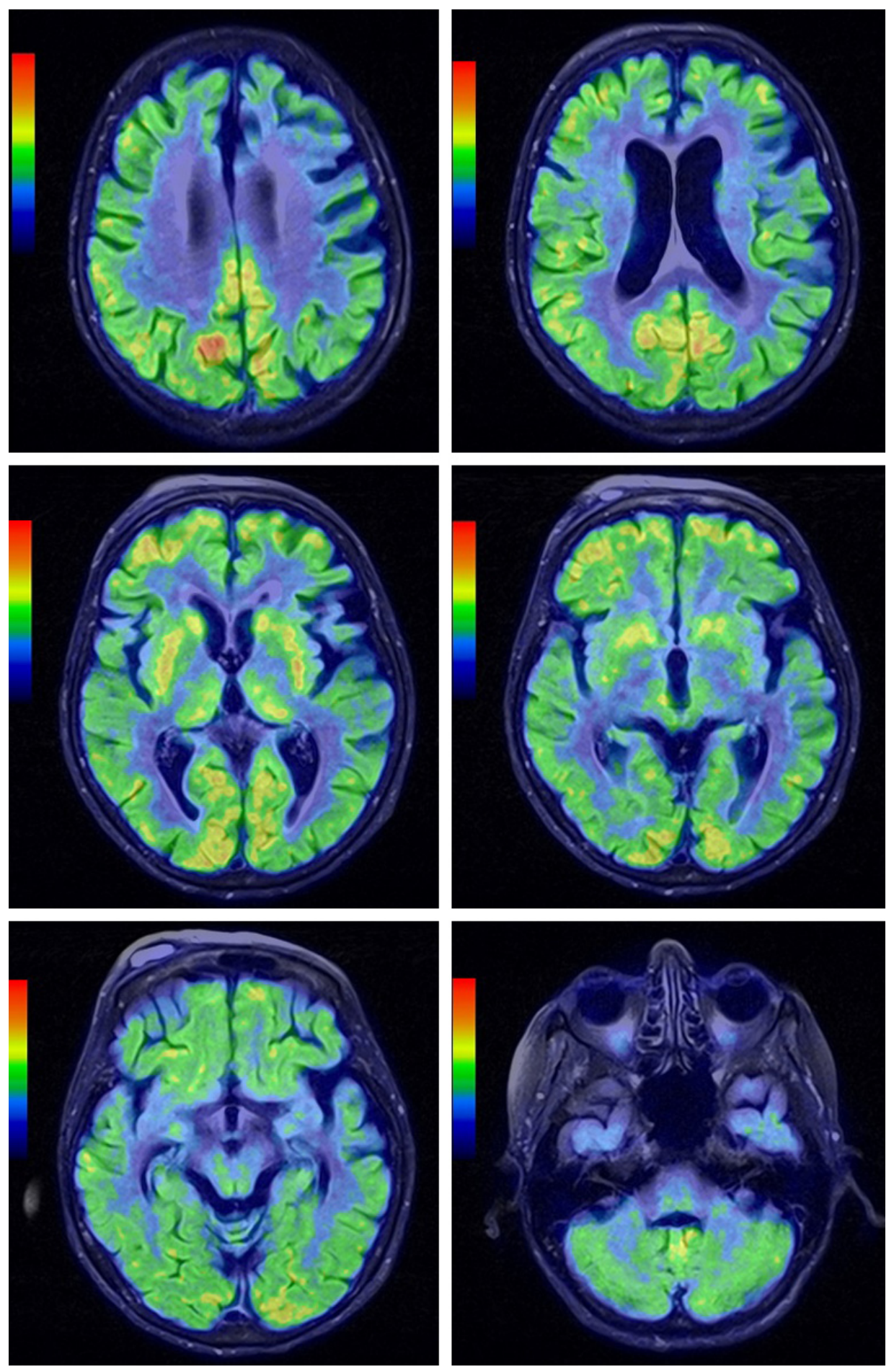

Figure 5 Coregistered PET and MR images in axial views in different levels demonstrating generalized brain atrophy, more prominent in the posterior frontal lobe, and hypometabolism of both cerebral hemispheres that was especially pronounced in the middle and posterior part of the left frontal lobe. Diffusely decreased metabolism was noted in the basal ganglia.

Abbreviations: PET, positron emission tomography; MR, magnetic resonance.

either condition. In our case, the patient had no PSP criteria of first level of certainty. The patient had no typical clinical symptoms for PSP, such as ocular muscle dysfunction, progressive gait freezing and nonfluent aphasia. Her symptoms did not fulfill the criteria for nonfluent/agrammatic, semantic or logopenic variant of primary progressive aphasia diagnoses. According to the new Movement Disorder Society (MDS) criteria, she had also one mandatory exclusion criterion for PSP that was prominent, confirmed and otherwise unexplained autonomic failure, which could cause the repeated falls. ${ }^{13}$ 
Furthermore, postural instability and falls developed only in the late stage of the disease. Although polysomnography testing has revealed the significantly high prevalence of RBD in MSA patients, ${ }^{2}$ it was not performed in our case. The patient had no clinical signs of sleep-related disorder, neither in the disease manifestation nor during the course of the disease; hence, we did not think it was necessary to perform a polysomnography test.

In conclusion, in our case, all neurological signs and symptoms as well as the disease course are more typical for MSA, despite the imaging findings and cognitive decline which developed in the late stage of the disease.

\section{Concluding remarks}

The diagnosis of MSA is primarily based on clinical criteria, although several diagnostic studies may be helpful to support the diagnosis or to rule out other diseases. Brain imaging techniques such as MRI and FDG-PET are frequently used and can be helpful, particularly in cases that present with atypical features.

Consensus-based diagnostic criteria for MSA have only been validated retrospectively. Symptoms and disease progression are still the most important in the differential diagnostic process because of the absence of reliable and widely available ante-mortem biomarkers. Future clinical research should thus attempt to define early MSA diagnosis through a combination of clinical, imaging and molecular markers.

\section{Disclosure}

The authors report no conflicts of interest in this work.

\section{References}

1. Gilman S, Wenning GK, Low PA, et al. Second consensus statement on the diagnosis of multiple system atrophy. Neurology. 2008;71(9): 670-676.

2. Palma JA, Fernandez-Cordon C, Coon EA, et al. Prevalence of REM sleep behavior disorder in multiple system atrophy: a multicentral study and meta-analysis. Clin Auton Res. 2015;25(1):69-75.

3. Koga S, Aoki N, Uitti RJ, et al. When DLB, PD, and PSP masquerade as MSA: an autopsy study of 134 patients. Neurology. 2015;85(5): 404-412.

4. Höglinger GU, Kassubek J, Csoti I, et al. Differentiation of atypical Parkinson syndromes. J Neural Transm (Vienna). 2017;124(8):997-1004.

5. Ubhi K, Low P, Maslish E. Multiple system atrophy: a clinical and neuropathological perspective. Trends Neurosci. 2011;34(11):581-590.

6. Bott NT, Radke A, Stephens ML, Kramer JH. Frontotemporal dementia: diagnosis, deficits and management. Neurodegener Dis Manag. 2014; 4(6):439-454.

7. Lee EA, Cho HI, Kim SS, Lee WY. Comparison of magnetic resonance imaging in subtypes of multiple system atrophy. Parkinsonism Relat Disord. 2004;10(6):363-368.

8. Teune LK, Bartels AL, de Jong BM, et al. Typical cerebral metabolic patterns in neurodegenerative brain diseases. Mov Disord. 2010; 25(14):2395-2404.

9. Ahmed Z, Asi YT, Sailer A, et al. The neuropathology, pathophysiology and genetics of multiple system atrophy. Neuropathol Appl Neurobiol. 2012;38(1):4-24.

10. Stamelou M, Bhatia KP. Atypical parkinsonism - new advances. Curr Opin Neurol. 2016;29(4):480-485.

11. Levin J, Kurz A, Arzberger T, Giese A, Höglinger GU. The differential diagnosis and treatment of atypical parkinsonism. Dtsch Arztebl Int. 2016;113(5):61-69.

12. Brown RG, Lacomblez L, Landwehrmeyer BG, et al; NNIPPS Study Group. Cognitive impairment in patients with multiple system atrophy and progressive supranuclear palsy. Brain. 2010;133(Pt 8): 2382-2393.

13. Höglinger GU, Respondek G, Stamelou M, et al; Movement Disorder Society-endorsed PSP Study Group. Clinical diagnosis pf progressive supranuclear palsy: the movement disorder society criteria. Mov Disord. 2017;32(6):853-864.
Neuropsychiatric Disease and Treatment

\section{Publish your work in this journal}

Neuropsychiatric Disease and Treatment is an international, peerreviewed journal of clinical therapeutics and pharmacology focusing on concise rapid reporting of clinical or pre-clinical studies on a range of neuropsychiatric and neurological disorders. This journal is indexed on PubMed Central, the 'PsycINFO' database and CAS,

\section{Dovepress}

and is the official journal of The International Neuropsychiatric Association (INA). The manuscript management system is completely online and includes a very quick and fair peer-review system, which is all easy to use. Visit http://www.dovepress.com/testimonials.php to read real quotes from published authors. 